\title{
Abdelhamid Mrabet, directeur général de WAFA Communication
}

Hugues Hotier

(2) OpenEdition

Journals

Édition électronique

URL : http://journals.openedition.org/communicationorganisation/1899

DOI : 10.4000/communicationorganisation.1899

ISSN : $1775-3546$

Éditeur

Presses universitaires de Bordeaux

Édition imprimée

Date de publication : 1 novembre 1996

ISSN : 1168-5549

\section{Référence électronique}

"Abdelhamid Mrabet, directeur général de WAFA Communication », Communication et organisation [En ligne], 10 | 1996, mis en ligne le 26 mars 2012, consulté le 20 avril 2019. URL : http:// journals.openedition.org/communicationorganisation/1899; DOI : 10.4000/

communicationorganisation. 1899

Ce document a été généré automatiquement le 20 avril 2019.

(C) Presses universitaires de Bordeaux 


\title{
Abdelhamid Mrabet, directeur général de WAFA Communication
}

\author{
Hugues Hotier
}

\section{NOTE DE L'ÉDITEUR}

Propos recueillis par Hugues Hotier

\section{NOTE DE L'AUTEUR}

Récemment nommé à la tête d'une filiale du groupe Wafabank spécialisée dans le crédit à la consommation, Abdelhamid Mrabet a géré Wafa Communication, à la fois direction de la communication du groupe et agence de communication et de mécénat. Il a été, deux mandats durant, président de l'Association des annonceurs du Maroc - ADAM (1991-1997). Abdelhamid Mrabet est considéré comme un des pionniers de la communication organisationnelle au Maroc. Hugues Hotier l'a rencontré à Casablanca et l'a invité à jeter un regard sur les quinze dernières années d'une vie professionnelle particulièrement riche.

1 Vous venez de changer de fonction en devenant directeur général d'une filiale du groupe Wafabank dont vous avez fondé et dirigé le service communication pendant quinze ans. Pouvez-vous nous retracer la naissance et l'histoire de cette direction ?

2 Au départ, au début des années 80 , il y eut la création d'une direction marketing. Cela n'existait pas, c'était tout nouveau dans la banque. Cette direction avait été confiée tout naturellement, par promotion interne, à un banquier. On a cherché dans le marché quelqu'un pour le seconder en tant que responsable du «marketing de la communication». J'ai été recruté pour cela, j'étais en quelque sorte le second du directeur marketing de la banque. Nous nous sommes très bien complétés, lui connaissant très bien la banque et moi connaissant le marketing de la communication. Au départ, la 
banque à laquelle j'appartiens était une petite banque avec une faible notoriété, bien qu'elle fût la plus ancienne.

3 Je saisis l'opportunité qui m'est offerte pour faire un peu d'histoire sur cette banque. Wafabank est la première banque moderne créée au Maroc, en 1904, par la Compagnie française de crédit et de banque à travers un établissement dénommé Compagnie algérienne domicilié à Alger, alors territoire français. Cette Compagnie algérienne de crédit et de banque s'était implantée à Tanger. Ce fut le premier organisme financier moderne du Maroc. Il n'a changé de dénomination que dans les années soixante, lorsqu'un groupe marocain en prit le contrôle. Ce groupe marocain était en fait un industriel marocain connu. Le groupe Suez comme partenaire étranger, le contrôle de la banque qui devint la Compagnie marocaine de crédit et de de banque, dénomination qui exista du milieu des années soixante jusqu'au milieu des années quatre-vingt. Pendant cette période, avec l'indépendance du Maroc, le monde bancaire se transforma considérablement : la plupart des autres banques se développèrent par fusion avec des compagnies étrangères. La Compagnie algérienne avait, au moment de l'indépendance, le plus important réseau au Maroc. Son identité était très forte.

4 L'arrivée d'un management marocain, à la fin des années soixante-dix, généra une politique de marketing importante afin de propulser la banque au niveau des grands établissements bancaires marocains. Cela a commencé par un marketing très offensif avec la création de produits nouveaux : carte de garantie de chèques, guichets automatiques, crédit revolving, assurance maladie pour les professions libérales, multirisques habitation... des produits qui ne furent généralisés par les autres banques que bien plus tard et dont nous avons été les précurseurs. Tout cela a renouvelé notre fonds de commerce, a développé notre notoriété et nous a attiré une clientèle de cadres et de professions libérales. Ce marketing très agressif eut pour effet de développer une image d'innovateurs et de trouble-fête. Mais nous avons observé que nous ne profitions pas assez de cette image parce que nous avions un problème d'identité. Compagnie marocaine de crédit et de banque, avec une traduction en arabe et deux sigles différents, cela était un peu compliqué. D'autant que c'était la même chose pour les autres banques - BMCI, BMCE - tout cela créait des confusions. Il fut donc décidé de changer de nom. Ce qui, en interne, fut une surprise pour tout le personnel à un moment où les esprits étaient occupés par les restructurations et l'innovation.

5 Je fus nommé responsable de ce projet. Nous y avons travaillé pendant près d'une année et demie pour arriver, le 1er janvier 1985, à une opération spectaculaire : transformer la CMCB en Wafabank. Je dis bien «spectaculaire " parce que nous avons bénéficié de tous les atouts. Dans cette affaire, nous avons été très professionnels et nous sommes allés jusqu'au bout de notre politique en changeant à la fois le nom, l'identité visuelle, les couleurs, les façades le 1er janvier et en faisant une politique de communication absolument globale. De la communication interne, avec plusieurs événements, des relations publiques avec des réceptions et des cocktails qui laissèrent un souvenir vivace aux participants. Imaginez un buffet somptueux reproduisant les lettres WAFA, le décor en vert et jaune, les hôtesses vêtues élégamment aux couleurs de la banque... Notre campagne a été très courte. Bénéficiant d'une période calme, elle a atteint ses objectifs en trois semaines. Ce changement de nom a été l'occasion de faire savoir, en interne comme en externe, qui nous étions. Nous avons édité des brochures que nous avons distribuées, et il y a donc eu un important volet informatif dans cette campagne. Histoire, valeurs, missions, produits... tout a été porté à la connaissance du public. Nous ne nous sommes 
pas contentés de devenir Wafabank, nous avons aussi défini le nouveau nom, nous avons capitalisé. Média peu employé à l'époque, les autobus arboraient une affiche avec le slogan « Hier CMCB., aujourd'hui Wafabank » avec la poignée de main qui est notre logo. Tout montrait une image de modernité. En fait, cette poignée de main était le seul héritage du passé, et constituait un logo nettement supérieur à tous ceux qui nous avaient été proposés. Nous l'avons restylisé, redessiné, encadré. Nous lui avons donné une force à travers les couleurs vert et jaune. Vert et jaune quand tout le monde était en bleu, c'était un facteur puissant de distinction et de lisibilité. Qui plus est, ces couleurs portent beaucoup de symboles. Quant au nom, Wafa (en arabe : fidélité), il était particulièrement bien trouvé : simple à retenir et porteur, il témoignait aussi de la fierté de sa marocanité que la banque ressent et qui est une de ses valeurs essentielles. N'oublions pas qu'à l'époque nous étions la seule banque appartenant à des Marocains, les autres étant majoritairement à capitaux étrangers. Il fallut attendre la "marocanisation » de 1973 pour que les banques marocaines aient $50 \%$ de capitaux marocains. Même aujourd'hui où nous avons mis en bourse une partie du capital, ce dernier est très majoritairement marocain.

\section{Les retombées en interne ont-elles été importantes?}

7 L'opération a été très valorisante pour l'ensemble du personnel. Nous avons fait faire une étude par un cabinet parisien, "Intelligence », et nous avons constaté que la valorisation avait été particulièrement forte pour l'encadrement. La période du changement a été euphorique. Le succès était d'autant plus grand que l'opération était risquée. Ya-t-il eu d'autres retombées?

9 C'est à partir de là qu'on a commencé à penser à la création d'un groupe Wafabank. Cela a été progressif. On a restructuré Wafabank en créant autour d'elle un ensemble de filiales satellisées de telle sorte que le client trouve à Wafabank une offre globale et harmonieuse. Il y a autre chose : avec ce changement de nom et la communication faite autour, on a propulsé la notoriété et l'image au-delà de ce que nous étions réellement. Le problème pour nous a alors été de hisser la banque au niveau de l'image et de la notoriété auxquelles elle était arrivée. Nous avions obtenu un taux d'attractivité et de Top of mind très fort. Il a fallu le capitaliser et, pour y répondre, élever les standards, mettre la banque à niveau dans tous les domaines: dans le domaine de l'informatique, dans le domaine du siège social, dans le domaine des ressources humaines. On s'est fait aider par les plus grands cabinets de consultants. Une réflexion a été menée dans tous les domaines pour améliorer la qualité et la compétitivité dans tous les domaines. Cela a été le chantier qui nous a occupés après le changement de nom. Dans le domaine de la communication, nous avons engagé des actions assez spectaculaires comme, par exemple, le parrainage d'un grand club marocain de football, le WAK. Cela a amplifié encore notre notoriété dans des milieux nouveaux.

11 Notre premier slogan était "Avec Wafabank, un nouveau style de banque », mais on ne peut pas utiliser le mot nouveau de façon durable, il fallait aussi personnifier et donner un contenu à notre style de banque. Aussi, quand on a créé l'offre globale du Groupe, on a employé un nouveau slogan: «Wafabank, jusqu'au bout de vos projets », signifiant ainsi que toutes les filiales concourent à amener le client jusqu'au bout de ses projets. Cette campagne a utilisé comme élément de communication le relais, la course de relais dans laquelle chaque filiale se transmet le témoin afin de conduire le client à la réussite. 
12 Après cela, nous avons engagé la banque vers la création de nouveaux métiers. Avant les autres, nous avons abordé de nouveaux métiers : notamment les métiers liés au marché des capitaux. Nous avons adopté une nouvelle stratégie dont la philosophie était la spécialisation et l'intégration. À chaque homme, un métier, dans lequel il doit témoigner d'un grand professionnalisme. Chacun doit connaittre mieux que les autres les ficelles de son métier. Quant à l'intégration, c'est évidemment la coordination au sein de directions de groupe, pour une meilleure synergie.

Notons que la communication financière a pris une place importante ces dernières années surtout depuis l'ouverture d'une partie du capital Wafabank. Lors de cette dernière importante opération d'émission en bourse nous étions dans l'incertitude total quant à son succès, au point qu'on avait prévu des modalités pour pallier une insuffisance de demandes. Or, la demande a couvert trois fois et demie l'offre. La communication financière que nous avons faite à cette occasion présentait avec conviction notre histoire, nos valeurs, notre culture, notre management à travers l'action de la banque. La Bourse juge sur un ensemble, elle évalue l'entreprise globalement. La campagne a utilisé essentiellement l'édition et la presse car la cible est relativement restreinte et on n'a pas besoin de média grand public. Notre communication financière est désormais dotée d'un budget et d'un plan. Il y a un service relations avec les actionnaires, l'assemblée générale est un vrai lieu de débat quand tant d'autres ne sont que des chambres d'enregistrement. Nous sommes allés aussi loin que possible dans la connaissance des actionnaires et pousser nos clients à devenir actionnaires, donnant de la réalité à l'expression « aller à sa banque ». L'actionnaire-client doit se sentir chez lui quand il vient à sa banque.

\section{De quand date la fondation Wafabank?}

Réponse : La Fondation a été créée en même temps que les filiales du groupe Wafabank, donc dans la deuxième moitié des années quatre-vingt. Son action a débuté à la fin de cette décennie. À mon avis, elle est l'un des instruments les plus structurés en matière de mécénat. Il y a deux ans, nous avons créé une sorte de filiale de la Fondation, spécialisée dans le caritatif qui a engagé une opération intitulée Solidarité-Ramadan, qui consiste à distribuer des repas pendant la période du Ramadan. Actuellement, elle engage une autre opération qui est le soutien scolaire aux enfants défavorisés. Nous allons débuter une troisième action qui consistera à établir des ateliers qui assureront une formation professionnelle à des femmes délaissées.

Quelles ont été les étapes de la mise en place de la direction de la communication ? Comment cette nouveauté dans l'organigramme a-t-elle été accueillie ?

L'évolution de l'organisation s'est faite de la façon suivante. On a commencé par créer une direction du marketing, puis on est passé par une direction du développement dans laquelle la communication était présente. Après cela, on a créé la direction de la communication. Je dis bien direction de la communication. J'insiste parce que qui dit direction dit participation à l'élaboration de la stratégie. Enfin, quand on a créé le groupe Wafabank, on a créé une direction de la communication du Groupe, chargée non seulement de la communication de Wafabank, mais aussi de la communication de la fondation Wafabank et de toutes les filiales. L'idée était de coordonner l'ensemble.

\section{Combien de personnes cela représente-t-il et quelles sont leurs fonctions?}

Une vingtaine de personnes. Le personnel a été recruté en interne ou sur le marché. Nous sommes une structure de gestion plus que de création. Pour la création, nous faisons appel à des agences, marocaines ou étrangères. Pour les grandes créations, les 
événements importants, cela ne peut pas être autrement. Il faut faire appel aux talents et les mettre en compétition. Il faut tenir compte aussi des spécialisations. Si vous lancez une campagne sur le marché des capitaux, il y a de telles spécificités qu'il faut absolument faire appel à une agence de communication financière. Tout le monde ne peut pas communiquer sur la bourse et les SICAV. Nous nous chargeons en interne de la gestion: achats d'espaces, production, livraison. Un groupe comme le nôtre consomme beaucoup d'édition: publi-reportages, plaquettes, brochures, notices de bourse... nous avons beaucoup à faire. Wafabank emploie 1600 personnes et le groupe, au total, 2500 .

Je suppose que la dimension stratégique se situe à votre niveau, au niveau du directeur de la communication. Comment $s$ 'articule-t-élle avec la stratégie de l'entreprise?

21 D'abord, par ma présence au comité de direction. Je suis ainsi au courant de tout ce qui se passe à la banque. À partir de là, je suis préparé à gérer les événements de la banque, qu'il s'agisse de relations publiques ou de crise.

Il y a quelque années, avec l'affirmation de nos valeurs, nous agacions beaucoup de gens qui nous percevaient comme des donneurs de leçon. Nous avons alors été victimes d'une rumeur malveillante. La gestion de cette crise a été menée dans l'esprit de la stratégie générale de Wafabank. Nous n'avons pas communiqué en externe, mais nous avons communiqué en interne. Et la rumeur est partie comme elle était venue.

Dans la stratégie de l'entreprise, il y a le respect des valeurs que nous avons rassemblées en un code porté à la connaissance de tout le personnel et que chacun s'engage à respecter. Ce code est interne, il n'est pas un argument de communication externe je vous l'ai déjà dit, il n'y a pas d'auto proclamation chez nous.

Pour ce qui est de la communication, comment se situe Wafabank par rapport aux autres banques?

Ce qui différencie Wafabank, c'est sans doute l'existence d'un code de communication dont la première règle est : ne jamais parler pour ne rien dire. Une parole de leader doit être rare. La deuxième règle est : jamais d'auto proclamation. On apprécie qu'on dise du bien de nous mais jamais nous n'en disons nous-mêmes. La troisième règle concerne le respect des principes de base de notre identité aussi bien visuelle que morale. Ce n'est pas la qualité d'une annonce ou d'une campagne qui nous distingue, c'est notre continuité, notre stabilité qui a fait la force de notre communication. Notre président ne parle que rarement, mais son discours est écouté, nos responsables ne parlent que rarement, les expositions de notre Fondation sont de qualité. C'est pour cela que nous sommes leaders. 